LA-UR-01-3442

Approved for public release; distribution is unlimited.

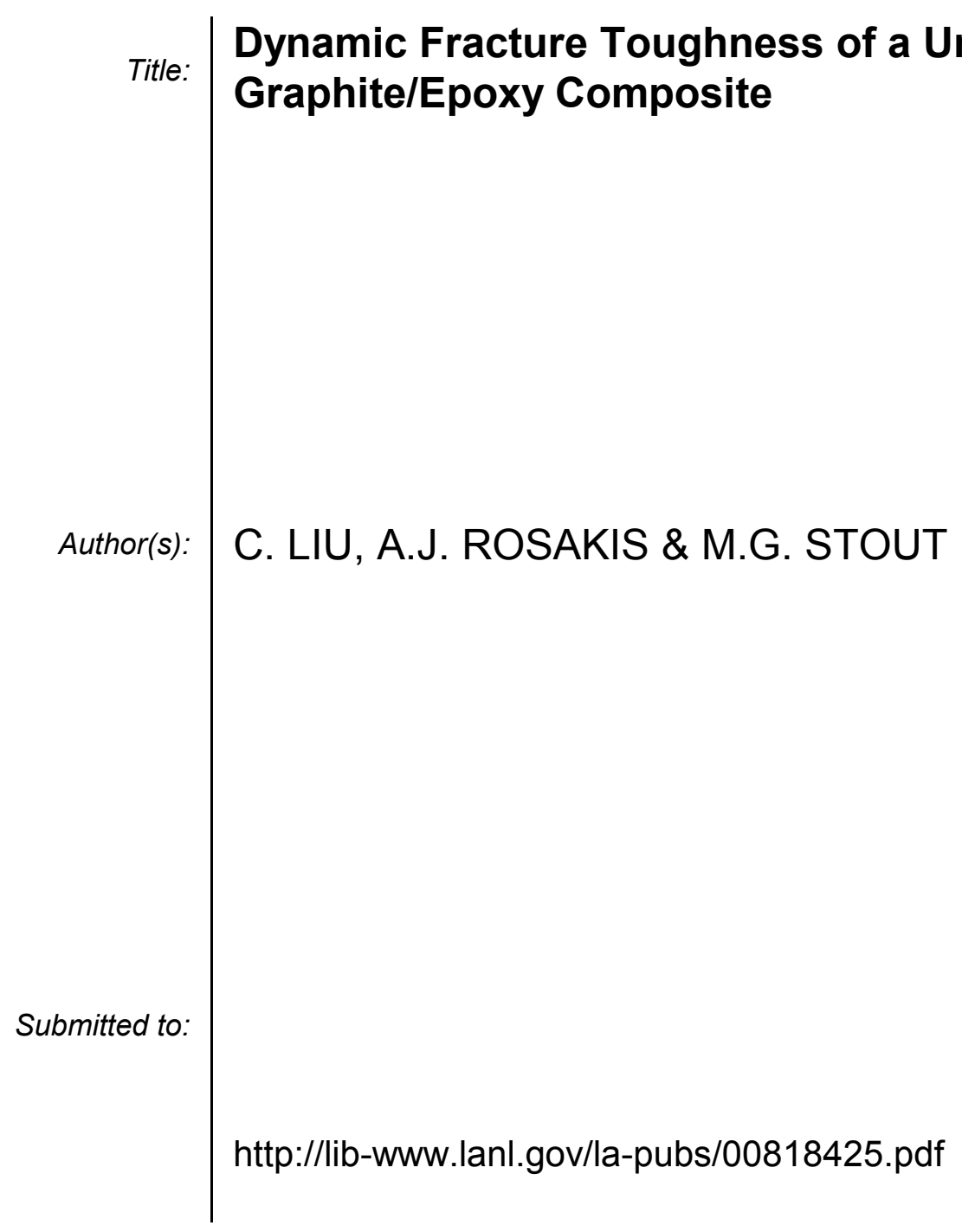

Los Alamos National Laboratory, an affirmative action/equal opportunity employer, is operated by the University of California for the U.S. Department of Energy under contract W-7405-ENG-36. By acceptance of this article, the publisher recognizes that the U.S. Government retains a nonexclusive, royaltyfree license to publish or reproduce the published form of this contribution, or to allow others to do so, for U.S. Government purposes. Los Alamos National Laboratory requests that the publisher identify this article as work performed under the auspices of the U.S. Department of Energy. Los Alamos National Laboratory strongly supports academic freedom and a researcher's right to publish; as an institution, however, the Laboratory does not endorse the viewpoint of a publication or guarantee its technical correctness. 


\title{
Dynamic Fracture Toughness of a Unidirectional Graphite/Epoxy Composite
}

\author{
C. LIU ${ }^{\dagger}$, A.J. RosaKIS ${ }^{\ddagger} \&$ M.G. STOUT ${ }^{\dagger}$ \\ ${ }^{\dagger}$ MST-8, Materials Science and Technology Division, Los Alamos National Laboratory \\ Los Alamos, New Mexico 87545, USA \\ ¥Graduate Aeronautical Laboratories, California Institute of Technology \\ Pasadena, California 91125, USA
}

\begin{abstract}
In this investigation, we studied the process of dynamic crack propagation in a fiber-reinforced composite material using the optical Coherent Gradient Sensing (CGS) technique combined with high-speed photography. The mode-I fracture toughness of the unidirectional graphite/epoxy composite, IM7/8551-7, as a function of the crack-tip speed, was measured quantitatively. It was found that up to the Rayleigh wave speed of the composite material, the mode-I fracture toughness is a decreasing function of the crack-tip velocity. This behavior is similar to that observed in the dynamic crack propagation along interfaces between two homogeneous solids.
\end{abstract}

\section{INTRODUCTION}

In recent years, we have seen the increasing use of fiber-reinforced composite materials in aeronautical, automotive, defense and other industries. The ever widen appreciation of fiber-reinforced composites is due to the fact that these materials have a very high stiffness to weight ratio. On the other hand, it has also been observed that crack-like defects develop easily in these solids even under normal service loading conditions and definitely during low-velocity impact events. Failure processes in these materials often initiate from such damage. To conclusively understand the mechanisms that control the fracture process in these materials, one must be able to experimentally measure the fracture toughness of fiber-reinforced composites subjected to different transient loading conditions, and to do so for both stationary and propagating cracks.

To date, most of the experimental measurements of composite fracture toughness are based on mechanical techniques, where far field loads and overall deformations are measured. The near-tip parameters that really control the fracture event are inferred through numerical calibration or other indirect analytical models and methods [1, 2, 3, 4]. For quasi-static loading and for stationary cracks, such techniques can provide useful information regarding the fracture resistance of the material. However, when the loading and subsequent crack growth take place in very short times, boundary value measurements are incapable of providing meaningful information regarding material's dynamic fracture behavior. The only alternative in such cases are optical methods that directly measure near-tip quantities when combined with appropriate high-speed photography methods.

Using a procedure for preparing optically flat and specularly reflective surfaces on composites, developed at the California Institute of Technology and at the Los Alamos National Laboratory, Liu et al. [5] studied the quasi-static fracture phenomenon in composite materials. In this investigation, we will experimentally study the dynamic fracture processes in a fiber-reinforced composite material using the optical method of Coherent Gradient Sensing (CGS). The deformation field surrounding the dynamically growing crack in the unidirectional fiber-reinforced composite is measured using high-speed photography. Based on an elastodynamic analysis of the deformation field near a steady-state propagating crack tip, in an orthotropic solid, the value of the dynamic stress-intensity factor can be extracted from the CGS fringe pattern at each moment. Finally, the relationship between the mode-I dynamic fracture toughness and the crack-tip velocity is established for the graphite/epoxy unidirectional fiber-reinforced composite, IM7/8551-7. 
DEFORMATION FIELD SURROUNDING THE TIP OF A GROWING CRACK IN ORTHOTROPIC SOLID

In order to interpret the CGS fringe patterns obtained from the experiment on the unidirectional fiberreinforced composite, an analytic expression of the deformation field surrounding the tip of a dynamically propagating crack in an orthotropic material is required. General results regarding dynamic crack growth in anisotropic materials have been discussed by $\mathrm{Wu}$ [6] and by Yang et al. [7]. In this section, we summarize the analytic results about the deformation field surrounding the tip of a dynamically propagating crack in an orthotropic material. Specifically, the leading terms that dominate the deformation field surrounding the crack tip are presented explicitly.

\section{Problem Description}

Consider the problem depicted in Figure 1, where a semi-infinite straight crack propagates dynamically in an orthotropic material. Here we only consider that the crack front, or edge, is a straight line parallel to the

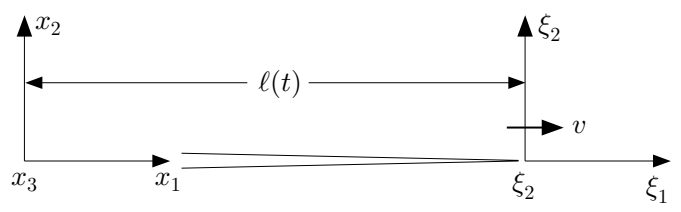

Figure 1: A semi-infinite crack propagates in an orthotropic solid.

$x_{3}$-axis. The coordinate $\left(x_{1}, x_{2}, x_{3}\right)$ is a fixed Cartesian system and the $x_{1}$-axis is pointing in the crack growth direction.

Suppose that the deformation is either plane strain or plane stress. The deformation field can be described by the two in-plane displacement components, $u_{1}\left(x_{1}, x_{2}, t\right)$ and $u_{2}\left(x_{1}, x_{2}, t\right)$, that are functions of the two inplane coordinates $x_{1}$ and $x_{2}$, and time $t$. Associated with this displacement field, we also have the stress and strain fields, $\sigma_{\alpha \beta}$ and $\epsilon_{\alpha \beta}(\alpha, \beta=1,2)$. For infinitesimal deformation, the relationship between the displacement and strain components is given by

$$
\epsilon_{\alpha \beta}\left(x_{1}, x_{2}, t\right)=\frac{1}{2}\left\{u_{\alpha, \beta}\left(x_{1}, x_{2}, t\right)+u_{\beta}, \alpha\left(x_{1}, x_{2}, t\right)\right\},
$$

and in the absence of body force density, the equation of motion for planar deformation can be written as

$$
\sigma_{\alpha \beta}, \beta\left(x_{1}, x_{2}, t\right)=\varrho \ddot{u}_{\alpha}\left(x_{1}, x_{2}, t\right),
$$

where $\varrho$ is the mass density of the material.

In this study, we only consider a homogeneous, linearly elastic, and orthotropic material. Meanwhile, $\left(x_{1}, x_{2}, x_{3}\right)$ is also the principal axes of the material. For such a material undergoing planar deformation, the generalized Hooke's law takes the form

$$
\epsilon_{11}=b_{11} \sigma_{11}+b_{12} \sigma_{22}, \quad \epsilon_{22}=b_{12} \sigma_{11}+b_{22} \sigma_{22}, \quad \epsilon_{12}=\frac{b_{66}}{2} \sigma_{12},
$$

where $b_{i j}$ are the elastic compliances of the material. In terms of the elastic moduli, $c_{i j}$, the generalized Hooke's law can be rewritten as

$$
\sigma_{11}=c_{11} \epsilon_{11}+c_{12} \epsilon_{22}, \quad \sigma_{22}=c_{12} \epsilon_{11}+c_{22} \epsilon_{22}, \quad \sigma_{12}=2 c_{66} \epsilon_{12} .
$$

Now, in terms of the displacement components $u_{\alpha}\left(x_{1}, x_{2}, t\right)$ and the elastic moduli $c_{i j}$, the equation of motion (2) can be rewritten as

$$
c_{11} u_{1,11}+c_{66} u_{1,22}+\left(c_{12}+c_{66}\right) u_{2}, 12=\varrho \ddot{u}_{1}, \quad c_{66} u_{2,11}+c_{22} u_{2,22}+\left(c_{12}+c_{66}\right) u_{1,12}=\varrho \ddot{u}_{2} .
$$

For planar deformations we may define three nondimensional material parameters in terms of $b_{i j}$ as follows:

$$
\lambda=\frac{b_{11}}{b_{22}}, \quad \rho=\frac{2 b_{12}+b_{66}}{2 \sqrt{b_{11} b_{22}}}, \quad \kappa=\frac{3 \sqrt{b_{11} b_{22}}+b_{12}}{\sqrt{b_{11} b_{22}}-b_{12}} .
$$


Notice that for isotropic materials, $\lambda$ and $\rho$ equal 1 , while $\kappa=(3-v) /(1+v)$ for plane-stress and $\kappa=3-4 v$ for plane-strain deformations respectively. In the degenerate case of an isotropic material, $v$ denotes Poisson's ratio. The positive definiteness of the strain energy density requires that

$$
b_{11}>0, \quad b_{11} b_{22}-b_{12}^{2}>0, \quad b_{66}>0 .
$$

In terms of $\lambda, \rho$, and $\kappa$, the above requirement becomes

$$
\lambda>0, \quad \rho>-1, \quad \text { and } \begin{cases}\kappa>1, & \text { for } \rho>1, \\ 1<\kappa<\frac{3+\rho}{1-\rho}, & \text { for }-1<\rho<1 .\end{cases}
$$

The dilatational wave speed along the $x_{1}$-direction, $c_{l}$, and the shear wave speed, $c_{s}$, are defined by the following,

$$
c_{l}=\left(\frac{c_{11}}{\varrho}\right)^{1 / 2}, \quad c_{s}=\left(\frac{c_{66}}{\varrho}\right)^{1 / 2} .
$$

Several parameters will also be used in the following derivations and they are

$$
\alpha_{l}^{2}(v)=1-\frac{v^{2}}{c_{l}^{2}}, \quad \alpha_{s}^{2}(v)=1-\frac{v^{2}}{c_{s}^{2}}, \quad \eta^{2}=\left(\frac{c_{l}}{c_{s}}\right)^{2}=\left(\frac{\kappa+1}{\kappa-1}\right) \frac{(3-\kappa)+\rho(\kappa+1)}{4 \sqrt{\lambda}},
$$

where $\alpha_{l}(v)$ and $\alpha_{s}(v)$ are functions of crack-tip speed, $v$, while $\eta$ is a material constant and for isotropic solids, $\eta^{2}=(\kappa+1) /(\kappa-1)$.

\section{Steady-State Crack Growth and Stroh Representation}

Because steady state conditions prevail very close to the moving crack tip, the three variables $\left(x_{1}, x_{2}, t\right)$ can be reduced to two, i.e.,

$$
u_{1}\left(x_{1}, x_{2}, t\right)=u_{1}\left(x_{1}-v t, x_{2}\right) \quad u_{2}\left(x_{1}, x_{2}, t\right)=u_{2}\left(x_{1}-v t, x_{2}\right) .
$$

According to Stroh [8], the displacement field in the moving coordinate $\left(\xi_{1}, \xi_{2}, \xi_{3}\right)$ can be expressed as

$$
\left.\begin{array}{l}
u_{1}=2 \operatorname{Re}\left\{A_{11} f_{1}\left(\xi_{1}+\mu_{1} \xi_{2}\right)+A_{12} f_{2}\left(\xi_{1}+\mu_{2} \xi_{2}\right)\right\} \\
u_{2}=2 \operatorname{Re}\left\{A_{21} f_{1}\left(\xi_{1}+\mu_{1} \xi_{2}\right)+A_{22} f_{2}\left(\xi_{1}+\mu_{2} \xi_{2}\right)\right\}
\end{array}\right\}
$$

where $f_{\alpha}(z)(\alpha=1,2)$ are two arbitrary analytic functions and $A_{\alpha \beta}$ and $\mu_{\alpha}$ are undetermined constants. In the above expression, $\operatorname{Re}\{\cdot\}$ represents the real part of any complex quantity. By using Stroh representation for the displacement components, (10), the stress field can be expressed in terms of $f_{\alpha}(z)$ as

$$
\left.\begin{array}{l}
\sigma_{11}=2 \operatorname{Re}\left\{\left(c_{11} A_{11}+2 c_{12} \mu_{1} A_{21}\right) f_{1}^{\prime}\left(\xi_{1}+\mu_{1} \xi_{2}\right)+\left(c_{11} A_{12}+2 c_{12} \mu_{2} A_{22}\right) f_{2}^{\prime}\left(\xi_{1}+\mu_{2} \xi_{2}\right)\right\} \\
\left.\sigma_{22}=2 \operatorname{Re}\left\{\left(c_{12} A_{11}+2 c_{22} \mu_{1} A_{21}\right) f_{1}^{\prime}\left(\xi_{1}+\mu_{1} \xi_{2}\right)+\left(c_{12} A_{12}+2 c_{22} \mu_{2} A_{22}\right) f_{2}^{\prime}\left(\xi_{1}+\mu_{2} \xi_{2}\right)\right\}\right\} . \\
\sigma_{12}=2 \operatorname{Re}\left\{c_{66}\left(A_{21}+2 \mu_{1} A_{11}\right) f_{1}^{\prime}\left(\xi_{1}+\mu_{1} \xi_{2}\right)+c_{66}\left(A_{22}+2 \mu_{2} A_{11}\right) f_{2}^{\prime}\left(\xi_{1}+\mu_{2} \xi_{2}\right)\right\}
\end{array}\right\} .
$$

For the existence of a nontrivial solution of the constants $A_{\alpha \beta}$, we have the following restriction on the parameter $\mu_{\alpha}(\alpha=1,2)$,

$$
\left(c_{11}+c_{66} \mu_{\alpha}^{2}-\varrho v^{2}\right)\left(c_{66}+c_{22} \mu_{\alpha}^{2}-\varrho v^{2}\right)-\mu_{\alpha}^{2}\left(c_{12}+c_{66}\right)^{2}=0 .
$$

By using the definitions given in (6) and (8), the above condition becomes

$$
\mu^{4}+\left\{\alpha_{l}^{2}(v) \eta^{2}+\frac{\alpha_{s}^{2}(v)}{\eta^{2} \lambda}-\frac{(1+\gamma)^{2}}{\eta^{2} \lambda}\right\} \mu^{2}+\frac{\alpha_{l}^{2}(v) \alpha_{s}^{2}(v)}{\lambda}=0, \quad \gamma=\sqrt{\lambda} \eta^{2}\left(\frac{3-\kappa}{1+\kappa}\right) .
$$

We have dropped the subscript of $\mu_{\alpha}$ in writing (13). In a more convenient form, (13) can be rewritten as

$$
\mu^{4}+2 s(v) \zeta(v) \mu^{2}+\zeta^{2}(v)=0, \quad s(v)=\frac{\lambda \eta^{4} \alpha_{l}^{2}(v)+\alpha_{s}^{2}(v)-(1+\gamma)^{2}}{2 \sqrt{\lambda} \eta^{2} \alpha_{l}(v) \alpha_{s}(v)}, \quad \zeta(v)=\frac{\alpha_{l}(v) \alpha_{s}(v)}{\sqrt{\lambda}} .
$$

Once again, $s(v)$ and $\zeta(v)$ are functions of the crack-tip speed, as well as the elastic constants of the orthotropic material. 


\section{Subsonic Crack Propagation}

When the crack-tip speed $v$ is in the range of $0<v<c_{s}<c_{l}$, the crack is said to propagate in the subsonic regime, in which we have

$$
\alpha_{l}^{2}(v)=1-\frac{v^{2}}{c_{l}^{2}}>0, \quad \text { and } \quad \alpha_{s}^{2}(v)=1-\frac{v^{2}}{c_{s}^{2}}>0,
$$

and $\alpha_{l}(v), \alpha_{s}(v), s(v)$, and $\zeta(v)$ are all real. We also have $\zeta(v)>0$ and $s(v)>-1$. One can show that equation (14) has no real solutions and the complex solutions that have positive imaginary parts are

$$
\mu_{\alpha}(v)=\left\{\begin{array}{ll}
i \sqrt{\zeta(v)}\left\{\sqrt{\frac{s(v)+1}{2} \pm \sqrt{\left.\frac{s(v)-1}{2}\right\},}},\right. & \text { for } s(v)>1, \\
\sqrt{\zeta(v)}\left\{ \pm \sqrt{\frac{1-s(v)}{2}}+i \sqrt{\frac{1+s(v)}{2}}\right\}, & \text { for }-1<s(v)<1,
\end{array} \quad(\alpha=1,2)\right.
$$

The choice of the positive imaginary part will ensure that the upper complex $\left(\xi_{1}+\mu_{\alpha} \xi_{2}\right)$-plane corresponds to the physical plane where $\xi_{2} \geqslant 0$. In (15), we spell out the fact that $\mu_{\alpha}$ depends on the crack-tip speed $v$.

With either solution for $\mu_{\alpha}(v)$ given in (15), the nontrivial solution for the coefficients $A_{\alpha \beta}$ can be obtained. One set of such solutions is

$$
A_{11}=1, \quad A_{21}=-\lambda_{1}(v), \quad A_{12}=-\frac{1}{\lambda_{2}(v)}, \quad A_{22}=1, \quad \lambda_{\alpha}(v)=\frac{\eta^{2} \alpha_{l}^{2}(v)+\mu_{\alpha}^{2}(v)}{(1+\gamma) \mu_{\alpha}(v)}, \quad \alpha=1,2 .
$$

Now, according to (10), the displacement field near the moving crack tip can be written as

$$
u_{1}=2 \operatorname{Re}\left\{f_{1}\left(z_{1}\right)-\frac{1}{\lambda_{2}} f_{2}\left(z_{2}\right)\right\}, \quad u_{2}=2 \operatorname{Re}\left\{-\lambda_{1} f_{1}\left(z_{1}\right)+f_{2}\left(z_{2}\right)\right\},
$$

and according to (11), the stress field near the moving crack tip can be similarly written as

$$
\left.\begin{array}{l}
\sigma_{11}=2 c_{66} \operatorname{Re}\left\{\left(\eta^{2}-\gamma \mu_{1} \lambda_{1}\right) f_{1}^{\prime}\left(z_{1}\right)-\frac{\eta^{2}-\gamma \mu_{2} \lambda_{2}}{\lambda_{2}} f_{2}^{\prime}\left(z_{2}\right)\right\} \\
\sigma_{22}=2 c_{66} \operatorname{Re}\left\{\left(\gamma-\lambda \eta^{2} \mu_{1} \lambda_{1}\right) f_{1}^{\prime}\left(z_{1}\right)-\frac{\gamma-\lambda \eta^{2} \mu_{2} \lambda_{2}}{\lambda_{2}} f_{2}^{\prime}\left(z_{2}\right)\right\} \\
\sigma_{12}=2 c_{66} \operatorname{Re}\left\{\left(\mu_{1}-\lambda_{1}\right) f_{1}^{\prime}\left(z_{1}\right)-\frac{\mu_{2}-\lambda_{2}}{\lambda_{2}} f_{2}^{\prime}\left(z_{2}\right)\right\}
\end{array}\right\},
$$

where $z_{\alpha}=\xi_{1}+\mu_{\alpha} \xi_{2}(\alpha=1,2)$. In order to determine the deformation field surrounding the moving crack tip, we need to determine the forms of the two analytical functions $f_{1}\left(z_{1}\right)$ and $f_{2}\left(z_{2}\right)$.

By assuming that crack surface remains traction free, the most general solution for $\boldsymbol{f}(z)=\left\{f_{1}(z), f_{2}(z)\right\}^{\top}$ can be obtained as

$$
\boldsymbol{f}^{\prime}(z)=\boldsymbol{L}^{-1}\left\{z^{-1 / 2} \boldsymbol{a}(z)+\boldsymbol{b}(z)\right\}, \quad \overline{\boldsymbol{f}}^{\prime}(z)=\overline{\boldsymbol{L}}^{-1}\left\{z^{-1 / 2} \boldsymbol{a}(z)-\boldsymbol{b}(z)\right\},
$$

where $\boldsymbol{a}(z)$ and $\boldsymbol{b}(z)$ are two arbitrary entire functions that satisfy the following requirement

$$
\overline{\boldsymbol{a}}(z)=\boldsymbol{a}(z), \quad \overline{\boldsymbol{b}}(z)=-\boldsymbol{b}(z) .
$$

The matrix $\boldsymbol{L}$ is defined through

$$
\boldsymbol{L}=c_{66}\left[\begin{array}{cc}
\mu_{1}-\lambda_{1} & -\frac{\mu_{2}-\lambda_{2}}{\lambda_{2}} \\
\gamma-\lambda \eta^{2} \mu_{1} \lambda_{1} & -\frac{\gamma-\lambda \eta^{2} \mu_{2} \lambda_{2}}{\lambda_{2}}
\end{array}\right] .
$$

The complete solution for $\boldsymbol{f}^{\prime}(z)$ can be obtained by expanding $\boldsymbol{a}(z)$ and $\boldsymbol{b}(z)$ into a Taylor series. However, if attention is focused in the region very close to the moving crack tip, we only need to consider the most 
singular term in the solution for $\boldsymbol{f}^{\prime}(z)$, i.e., $\boldsymbol{f}^{\prime}(z)=\boldsymbol{L}^{-1} \boldsymbol{a}_{0} / \sqrt{z}$, where $\boldsymbol{a}_{0}$ is a real vector. By definition, the dynamic mode-I and mode-II stress-intensity factors, $K_{\mathrm{I}}$ and $K_{\mathrm{II}}$, at the moving crack tip, are related to the traction vector, $\left\{\sigma_{12}\left(\xi_{1}, 0\right), \sigma_{22}\left(\xi_{1}, 0\right)\right\}$ ahead of the moving crack tip, through

$$
\boldsymbol{k} \equiv\left\{K_{\mathrm{II}}, K_{\mathrm{I}}\right\}^{\top}=\lim _{\xi_{1} \rightarrow 0^{+}} \sqrt{2 \pi \xi_{1}}\left\{\sigma_{12}\left(\xi_{1}, 0\right), \sigma_{22}\left(\xi_{1}, 0\right)\right\}^{\top} .
$$

As a result, $\boldsymbol{a}_{0}=\boldsymbol{k} /(2 \sqrt{2 \pi})$ and finally, the most singular solutions for the two analytic functions $f_{1}^{\prime}\left(z_{1}\right)$ and $f_{2}^{\prime}\left(z_{2}\right)$ are determined to be

$$
\begin{aligned}
& f_{1}^{\prime}\left(z_{1}\right)=\frac{1}{2 c_{66} R(v)}\left(\frac{\mu_{2}-\lambda_{2}}{\lambda_{1}-\lambda_{2}} \cdot \frac{K_{\mathrm{I}}}{\sqrt{2 \pi}}-\frac{\gamma-\lambda \eta^{2} \mu_{2} \lambda_{2}}{\lambda_{1}-\lambda_{2}} \cdot \frac{K_{\mathrm{II}}}{\sqrt{2 \pi}}\right) \frac{1}{\sqrt{z_{1}}} \\
& \left.f_{2}^{\prime}\left(z_{2}\right)=\frac{\lambda_{2}}{2 c_{66} R(v)}\left(\frac{\mu_{1}-\lambda_{1}}{\lambda_{1}-\lambda_{2}} \cdot \frac{K_{\mathrm{I}}}{\sqrt{2 \pi}}-\frac{\gamma-\lambda \eta^{2} \mu_{1} \lambda_{1}}{\lambda_{1}-\lambda_{2}} \cdot \frac{K_{\mathrm{II}}}{\sqrt{2 \pi}}\right) \frac{1}{\sqrt{z_{2}}}\right),
\end{aligned}
$$

where $R(v)$ is the generalized Rayleigh wave function given by

$$
R(v)=\sqrt{\lambda} \eta^{2} \alpha_{l}(v) \alpha_{s}(v)-\frac{\sqrt{\lambda} \eta^{2} \alpha_{l}(v)+\gamma^{2} \alpha_{s}(v)}{\sqrt{\lambda} \eta^{2} \alpha_{l}(v)+\alpha_{s}(v)} .
$$

The Rayleigh wave speed of the orthotropic solid, $c_{\mathrm{R}}$, is such that $R\left(c_{\mathrm{R}}\right)=0$. One can show that for isotropic materials, $R(v)$ degenerates to the following,

$$
R(v)=\frac{D(v)}{1-\alpha_{l}(v) \alpha_{s}(v)}, \quad D(v)=4 \alpha_{l}(v) \alpha_{s}(v)-\left\{1+\alpha_{s}^{2}(v)\right\}^{2} .
$$

The displacement field and the stress field surrounding the moving crack tip are summarized in the following,

$$
\left.\begin{array}{rl}
u_{1}=\frac{2}{c_{66} R(v)} \operatorname{Re}\left\{\left(\frac{\mu_{2}-\lambda_{2}}{\lambda_{1}-\lambda_{2}} \sqrt{\frac{z_{1}}{2 \pi}}-\frac{\mu_{1}-\lambda_{1}}{\lambda_{1}-\lambda_{2}} \sqrt{\frac{z_{2}}{2 \pi}}\right) K_{\mathrm{I}}\right. \\
\left.-\left(\frac{\gamma-\lambda \eta^{2} \mu_{2} \lambda_{2}}{\lambda_{1}-\lambda_{2}} \sqrt{\frac{z_{1}}{2 \pi}}-\frac{\gamma-\lambda \eta^{2} \mu_{1} \lambda_{1}}{\lambda_{1}-\lambda_{2}} \sqrt{\frac{z_{2}}{2 \pi}}\right) K_{\mathrm{II}}\right\} \\
u_{2}=\frac{2}{c_{66} R(v)} \operatorname{Re}\{ & -\left(\lambda_{1} \frac{\mu_{2}-\lambda_{2}}{\lambda_{1}-\lambda_{2}} \sqrt{\frac{z_{1}}{2 \pi}}-\lambda_{2} \frac{\mu_{1}-\lambda_{1}}{\lambda_{1}-\lambda_{2}} \sqrt{\frac{z_{2}}{2 \pi}}\right) K_{\mathrm{I}} \\
& \left.+\left(\lambda_{1} \frac{\gamma-\lambda \eta^{2} \mu_{2} \lambda_{2}}{\lambda_{1}-\lambda_{2}} \sqrt{\frac{z_{1}}{2 \pi}}-\lambda_{2} \frac{\gamma-\lambda \eta^{2} \mu_{1} \lambda_{1}}{\lambda_{1}-\lambda_{2}} \sqrt{\frac{z_{2}}{2 \pi}}\right) K_{\mathrm{II}}\right\}
\end{array}\right\},
$$

and

$$
\left.\begin{array}{c}
\sigma_{11}=\frac{1}{R(v)} \operatorname{Re}\left\{\left(\frac{\mu_{2}-\lambda_{2}}{\lambda_{1}-\lambda_{2}} \cdot \frac{\eta^{2}-\gamma \mu_{1} \lambda_{1}}{\sqrt{2 \pi z_{1}}}-\frac{\mu_{1}-\lambda_{1}}{\lambda_{1}-\lambda_{2}} \cdot \frac{\eta^{2}-\gamma \mu_{2} \lambda_{2}}{\sqrt{2 \pi z_{2}}}\right) K_{\mathrm{I}}\right. \\
\left.-\left(\frac{\gamma-\lambda \eta^{2} \mu_{2} \lambda_{2}}{\lambda_{1}-\lambda_{2}} \cdot \frac{\eta^{2}-\gamma \mu_{1} \lambda_{1}}{\sqrt{2 \pi z_{1}}}-\frac{\gamma-\lambda \eta^{2} \mu_{1} \lambda_{1}}{\lambda_{1}-\lambda_{2}} \cdot \frac{\eta^{2}-\gamma \mu_{2} \lambda_{2}}{\sqrt{2 \pi z_{2}}}\right) K_{\mathrm{II}}\right\}, \\
\sigma_{22}=\frac{1}{R(v)} \operatorname{Re}\left\{\left(\frac{\mu_{2}-\lambda_{2}}{\lambda_{1}-\lambda_{2}} \cdot \frac{\gamma-\lambda \eta^{2} \mu_{1} \lambda_{1}}{\sqrt{2 \pi z_{1}}}-\frac{\mu_{1}-\lambda_{1}}{\lambda_{1}-\lambda_{2}} \cdot \frac{\gamma-\lambda \eta^{2} \mu_{2} \lambda_{2}}{\sqrt{2 \pi z_{2}}}\right) K_{\mathrm{I}}\right. \\
\left.-\left(\frac{\gamma-\lambda \eta^{2} \mu_{2} \lambda_{2}}{\lambda_{1}-\lambda_{2}} \cdot \frac{\gamma-\lambda \eta^{2} \mu_{1} \lambda_{1}}{\sqrt{2 \pi z_{1}}}-\frac{\gamma-\lambda \eta^{2} \mu_{1} \lambda_{1}}{\lambda_{1}-\lambda_{2}} \cdot \frac{\gamma-\lambda \eta^{2} \mu_{2} \lambda_{2}}{\sqrt{2 \pi z_{2}}}\right) K_{\mathrm{II}}\right\} \\
\sigma_{12}=\frac{1}{R(v)} \operatorname{Re}\left\{\left(\frac{\mu_{2}-\lambda_{2}}{\lambda_{1}-\lambda_{2}} \cdot \frac{\mu_{1}-\lambda_{1}}{\sqrt{2 \pi z_{1}}}-\frac{\mu_{1}-\lambda_{1}}{\lambda_{1}-\lambda_{2}} \cdot \frac{\mu_{2}-\lambda_{2}}{\sqrt{2 \pi z_{2}}}\right) K_{\mathrm{I}}\right. \\
\left.-\left(\frac{\gamma-\lambda \eta^{2} \mu_{2} \lambda_{2}}{\lambda_{1}-\lambda_{2}} \cdot \frac{\mu_{1}-\lambda_{1}}{\sqrt{2 \pi z_{1}}}-\frac{\gamma-\lambda \eta^{2} \mu_{1} \lambda_{1}}{\lambda_{1}-\lambda_{2}} \cdot \frac{\mu_{2}-\lambda_{2}}{\sqrt{2 \pi z_{2}}}\right) K_{\mathrm{II}}\right\}
\end{array}\right\} .
$$


Another crack-tip parameter is the energy release rate $\mathcal{G}$. By using Irwin's closure integral [9], the energy release rate $\mathcal{G}$ can be evaluated by

$$
\mathcal{G}=\lim _{\Delta a \rightarrow 0} \frac{1}{2 \Delta a} \int_{0}^{\Delta a}\left\{\sigma_{12}(x, 0) \delta_{1}(x, 0)+\sigma_{22}(x, 0) \delta_{2}(x, 0)\right\} \mathrm{d} x,
$$

where $\delta_{\alpha}(x, 0)=u_{\alpha}\left(x-\Delta a, 0^{+}\right)-u_{\alpha}\left(x-\Delta a, 0^{-}\right)(\alpha=1,2$ and $0 \leqslant x \leqslant \Delta a)$ is the crack-opening displacement. In terms of the two stress-intensity factors, $K_{\mathrm{I}}$ and $K_{\mathrm{II}}$, the energy release rate $\mathcal{G}$ can be expressed as

$$
\mathcal{G}=\frac{\eta}{2 c_{66} R(v)}\left\{1-\left(\frac{1+\gamma}{\sqrt{\lambda} \eta^{2} \alpha_{l}(v)+\alpha_{s}(v)}\right)^{2}\right\}^{1 / 2}\left(\alpha_{l}(v) K_{\mathrm{I}}^{2}+\sqrt{\lambda} \alpha_{s}(v) K_{\mathrm{II}}^{2}\right) .
$$

Once again, one can show that for isotropic solids, energy release rate $\mathcal{G}$ in (28) reduces to

$$
\mathcal{G}=\frac{1-\alpha_{s}^{2}}{2 c_{66} D}\left(\alpha_{l} K_{\mathrm{I}}^{2}+\alpha_{s} K_{\mathrm{II}}^{2}\right), \quad D=4 \alpha_{l} \alpha_{s}-\left(1+\alpha_{s}^{2}\right)^{2},
$$

which is the same as that given in Freund [10]. On the other hand, for a stationary crack in an orthotropic solid, one can show that the energy release rate $\mathcal{G}$ in (28) becomes

$$
\mathcal{G}=b_{11} \sqrt{\frac{1+\rho}{2}}\left(\lambda^{-3 / 4} K_{\mathrm{I}}+\lambda^{-1 / 4} K_{\mathrm{II}}\right)
$$

This expression is the same as that in Hutchinson and Suo [11].

\section{CGS TECHNIQUE AND ITS APPLICATION TO DYNAMIC FRACTURE OF COMPOSITES}

CGS, or the Coherent Gradient Sensing method is a full field, lateral shearing interferometric technique with an on-line filter. This method, when used in a reflective mode, measures the in-plane gradients of out-of-plane surface displacements around the crack tip. The basic governing equations of the method for a reflective setup, are the following [12],

$$
\frac{\partial u_{3}\left(\xi_{1}, \xi_{2}\right)}{\partial \xi_{1}}=\frac{n_{1} p}{2 \Delta}, \quad \frac{\partial u_{3}\left(\xi_{1}, \xi_{2}\right)}{\partial \xi_{2}}=\frac{n_{2} p}{2 \Delta},
$$

where $u_{3}\left(\xi_{1}, \xi_{2}\right)$ is the out-of-plane displacement of the specimen surface. Parameters $p$ and $\Delta$ are the pitch and separation of the two high density gratings, and finally, $n_{1}$ and $n_{2}$ are the fringe orders for the $\xi_{1}, \xi_{2}$ gradient contours respectively. Therefore, each CGS fringe is a locus of points that have the same slope in either $\xi_{1}$ - or $\xi_{2}$-direction, depending on the orientation of the two high density gratings.

For an isotropic solid subjected to plane stress deformation, the out-of-plane displacement $u_{3}\left(\xi_{1}, \xi_{2}\right)$ can directly be related to the first stress invariant. However, for anisotropic materials, the relation is not that simple. For orthotropic solid, according to the constitutive relation,

$$
\epsilon_{33}=b_{31} \sigma_{11}+b_{32} \sigma_{22}+b_{33} \sigma_{33},
$$

where $b_{31}, b_{32}$, and $b_{33}$ are elastic constants. For plane stress deformation, $\sigma_{33}=0$. Also, according to the generalized plane stress conditions, the out-of-plane surface displacement $u_{3}\left(\xi_{1}, \xi_{2}\right)$ can be related to the in-plane stress components through

$$
u_{3}\left(\xi_{1}, \xi_{2}\right)=\frac{h}{2}\left\{b_{31} \sigma_{11}\left(\xi_{1}, \xi_{2}\right)+b_{32} \sigma_{22}\left(\xi_{1}, \xi_{2}\right)\right\} .
$$

By using the stress field near the moving crack tip, the most singular term for the out-of-plane surface displacement can be expressed in terms of the dynamic stress-intensity factors by

$$
\begin{aligned}
u_{3}=\frac{h}{2 R(v)} \operatorname{Re}\left\{\left(\frac{\mu_{2}-\lambda_{2}}{\lambda_{1}-\lambda_{2}} \cdot \frac{\beta_{1}-\beta_{2} \mu_{1} \lambda_{1}}{\sqrt{2 \pi z_{1}}}-\frac{\mu_{1}-\lambda_{1}}{\lambda_{1}-\lambda_{2}} \cdot \frac{\beta_{1}-\beta_{2} \mu_{2} \lambda_{2}}{\sqrt{2 \pi z_{2}}}\right) K_{\mathrm{I}}\right. \\
\left.\quad-\left(\frac{\gamma-\lambda \eta^{2} \mu_{2} \lambda_{2}}{\lambda_{1}-\lambda_{2}} \cdot \frac{\beta_{1}-\beta_{2} \mu_{1} \lambda_{1}}{\sqrt{2 \pi z_{1}}}-\frac{\gamma-\lambda \eta^{2} \mu_{1} \lambda_{1}}{\lambda_{1}-\lambda_{2}} \cdot \frac{\beta_{1}-\beta_{2} \mu_{2} \lambda_{2}}{\sqrt{2 \pi z_{2}}}\right) K_{\mathrm{II}}\right\},
\end{aligned}
$$


where $\beta_{1}=b_{31} \eta^{2}+b_{32} \gamma$ and $\beta_{2}=b_{31} \gamma+b_{32} \lambda \eta^{2}$. Note that $\beta_{1}$ and $\beta_{2}$ are two material constants. The governing equation for the CGS technique, (31), would thus become

$$
F_{11}\left(\xi_{1}, \xi_{2}\right) K_{\mathrm{I}}+F_{12}\left(\xi_{1}, \xi_{2}\right) K_{\mathrm{II}}=n_{1}\left(\frac{p}{2 \Delta}\right), \quad F_{21}\left(\xi_{1}, \xi_{2}\right) K_{\mathrm{I}}+F_{22}\left(\xi_{1}, \xi_{2}\right) K_{\mathrm{II}}=n_{2}\left(\frac{p}{2 \Delta}\right),
$$

where

$$
\begin{aligned}
& F_{11}=-\frac{h}{4 \sqrt{2 \pi} R(v)} \operatorname{Re}\left\{\frac{\mu_{2}-\lambda_{2}}{\lambda_{1}-\lambda_{2}} \cdot \frac{\beta_{1}-\beta_{2} \mu_{1} \lambda_{1}}{\left(\xi_{1}+\mu_{1} \xi_{2}\right)^{3 / 2}}-\frac{\mu_{1}-\lambda_{1}}{\lambda_{1}-\lambda_{2}} \cdot \frac{\beta_{1}-\beta_{2} \mu_{2} \lambda_{2}}{\left(\xi_{1}+\mu_{2} \xi_{2}\right)^{3 / 2}}\right\}, \\
& F_{12}=\frac{h}{4 \sqrt{2 \pi} R(v)} \operatorname{Re}\left\{\frac{\gamma-\lambda \eta^{2} \mu_{2} \lambda_{2}}{\lambda_{1}-\lambda_{2}} \cdot \frac{\beta_{1}-\beta_{2} \mu_{1} \lambda_{1}}{\left(\xi_{1}+\mu_{1} \xi_{2}\right)^{3 / 2}}-\frac{\gamma-\lambda \eta^{2} \mu_{1} \lambda_{1}}{\lambda_{1}-\lambda_{2}} \cdot \frac{\beta_{1}-\beta_{2} \mu_{2} \lambda_{2}}{\left(\xi_{1}+\mu_{2} \xi_{2}\right)^{3 / 2}}\right\}, \\
& F_{21}=-\frac{h}{4 \sqrt{2 \pi} R(v)} \operatorname{Re}\left\{\frac{\mu_{1}\left(\mu_{2}-\lambda_{2}\right)}{\lambda_{1}-\lambda_{2}} \cdot \frac{\beta_{1}-\beta_{2} \mu_{1} \lambda_{1}}{\left(\xi_{1}+\mu_{1} \xi_{2}\right)^{3 / 2}}-\frac{\mu_{2}\left(\mu_{1}-\lambda_{1}\right)}{\lambda_{1}-\lambda_{2}} \cdot \frac{\beta_{1}-\beta_{2} \mu_{2} \lambda_{2}}{\left(\xi_{1}+\mu_{2} \xi_{2}\right)^{3 / 2}}\right\}, \\
& F_{22}=\frac{h}{4 \sqrt{2 \pi} R(v)} \operatorname{Re}\left\{\frac{\mu_{1}\left(\gamma-\lambda \eta^{2} \mu_{2} \lambda_{2}\right)}{\lambda_{1}-\lambda_{2}} \cdot \frac{\beta_{1}-\beta_{2} \mu_{1} \lambda_{1}}{\left(\xi_{1}+\mu_{1} \xi_{2}\right)^{3 / 2}}-\frac{\mu_{2}\left(\gamma-\lambda \eta^{2} \mu_{1} \lambda_{1}\right)}{\lambda_{1}-\lambda_{2}} \cdot \frac{\beta_{1}-\beta_{2} \mu_{2} \lambda_{2}}{\left(\xi_{1}+\mu_{2} \xi_{2}\right)^{3 / 2}}\right\} .
\end{aligned}
$$

If one knows the CGS fringe orders, $n_{1}$ or $n_{2}$, and the coordinates of the point $\left(\xi_{1}, \xi_{2}\right)$ on the fringes, the dynamic stress-intensity factors, $K_{\mathrm{I}}$ and $K_{\mathrm{II}}$, can be calculated using either of the equations in (35).

\section{MEASURING THE DYNAMIC FRACTURE TOUGHNESS OF THE UNIDIRECTIONAL FIBER-REINFORCED IM7/8551-7 GRAPHITE/EPOXY COMPOSITE}

\section{Composite Material, Specimen Preparation, and Experimental Procedure}

A unidirectional graphite/epoxy composite, IM7/8551-7, was used in this study. The microstructure of the unidirectional composite is shown in Figure 2, a collection of optical micrographs showing the three orthogonal material orientations. The diameter of the continuous fibers is approximately $5 \mu \mathrm{m}$ and the thickness of a laminate is on the order of $100 \mu \mathrm{m}$. The fiber volume fraction is approximately $60 \%$; it might be noted that the matrix is a rubber-toughened (mean particle size $10-75 \mu \mathrm{m}$ ) epoxy.

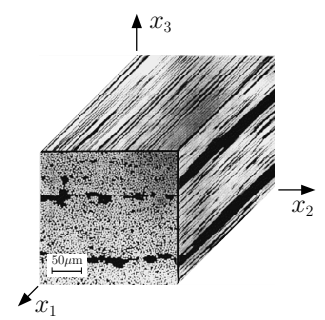

Figure 2: Micrographs of the graphite/epoxy unidirectional fiber-reinforced composite, IM7/8551-7.

A Cartesian coordinate system has been chosen such that the $x_{1}$-axis is along the fiber direction, the $x_{2}$ axis lies in the laminate plane and normal to the fibers, and the $x_{3}$-axis is normal to the laminate plane. Since the material, as shown in Figure 2, is symmetric with respect to the three coordinate planes it can be modeled as an orthotropic solid, and $\left(x_{1}, x_{2}, x_{3}\right)$ are the principal axes of the material. The elastic constants of the

Table 1: Material Elastic Constants

\begin{tabular}{lccc}
\hline \multirow{2}{*}{ Young's Moduli (GPa) } & $E_{11}$ & $E_{22}$ & $E_{33}$ \\
\cline { 2 - 4 } & 148.60 & 8.40 & 8.28 \\
\hline \hline \multirow{2}{*}{ Shear Moduli (GPa) } & $\mu_{12}$ & $\mu_{23}$ & $\mu_{13}$ \\
\cline { 2 - 4 } & 5.45 & 3.03 & 4.34 \\
\hline \hline \multirow{2}{*}{ Poisson's Ratios } & $v_{12}$ & $v_{23}$ & $v_{13}$ \\
\cline { 2 - 4 } & 0.32 & 0.37 & 0.35 \\
\hline
\end{tabular}

unidirectional graphite/epoxy composite have been measured with respect to the principal axes $\left(x_{1}, x_{2}, x_{3}\right)$, and the results are listed in Table 1. 
The specimen geometry we chose for this experimental study, was the single-edge-crack specimen as shown in Figure 3. The nominal dimensions of the specimen, shown in Figure 3, are: $2 l=150 \mathrm{~mm}, b=$

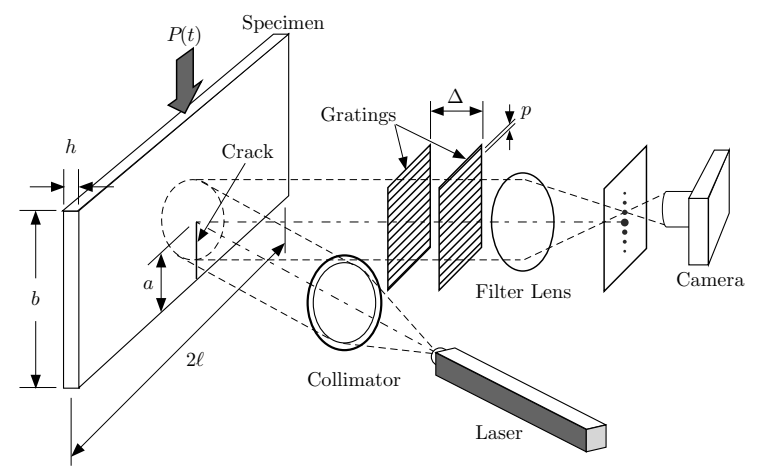

Figure 3: Schematic of CGS setup and single-edge-crack specimen.

$75 \mathrm{~mm}, h=6.5 \mathrm{~mm}$, and $a=25 \mathrm{~mm}$. In this experimental study, we concentrate on the situation where the crack is parallel to the fibers and the specimen plane is parallel to the laminates. A Cartesian coordinate system is chosen with its origin located at the crack tip. The axes of this system are aligned with the principal axes of the orthotropic material as shown in Figure 2, so that the deformation plane is the $\left(x_{1}, x_{2}\right)$-plane, with crack propagating along the $x_{1}$-axis. In this plane, the elastic compliances are listed in Table 2 . Based on

Table 2: Material Elastic Compliance

\begin{tabular}{cccccc}
\hline$b_{11}\left(\mathrm{GPa}^{-1}\right)$ & $b_{22}\left(\mathrm{GPa}^{-1}\right)$ & $b_{12}\left(\mathrm{GPa}^{-1}\right)$ & $b_{66}\left(\mathrm{GPa}^{-1}\right)$ & $b_{31}\left(\mathrm{GPa}^{-1}\right)$ & $b_{32}\left(\mathrm{GPa}^{-1}\right)$ \\
\hline $6.73 \times 10^{-3}$ & $1.19 \times 10^{-1}$ & $-2.15 \times 10^{-3}$ & $1.83 \times 10^{-1}$ & $-2.36 \times 10^{-3}$ & $-4.40 \times 10^{-2}$ \\
\hline
\end{tabular}

these values, the three nondimensional material parameters can be calculated to be $\lambda=0.0565, \rho=3.1676$, and $\kappa=2.7172$, respectively. Meanwhile, the dilatational wave speed along the fiber ( $x_{1}$-axis), $c_{l}$, the shear wave speed in the $\left(x_{1}, x_{2}\right)$-plane, and the Rayleigh wave speed on a surface parallel to the $\left(x_{1}, x_{3}\right)$-plane and in the direction of fiber ( $x_{1}$-axis), $c_{\mathrm{R}}$, are given in Table 3 .

Table 3: Characteristic Wave Speeds

\begin{tabular}{ccc}
\hline$c_{l}(\mathrm{~m} / \mathrm{sec})$ & $c_{s}(\mathrm{~m} / \mathrm{sec})$ & $c_{\mathrm{R}}(\mathrm{m} / \mathrm{sec})$ \\
\hline 9819.84 & 1874.62 & 1852.50 \\
\hline
\end{tabular}

A very high quality surface preparation is required in order to apply the CGS technique to composite materials. However, because of the microscopic nature of the composite, we cannot polish the specimen surface to make it optically flat and reflective, nor can we directly deposit a reflective aluminum film on the specimen by vacuum deposition. Instead, the following procedure was developed and used. An optically flat glass plate is coated with a thin aluminum film, having a thickness of only several angstroms. A layer of segregation material is intentionally maintained between the coating and the glass, to prevent strong adhesion between the aluminum film and the glass. This material was the residue of liquid soap used to clean the glass. The coated glass is then affixed to the sample using an epoxy adhesive, which glues the coated surface of the optically flat glass to the sample. The epoxy adhesive was a PC-1 Bipax of epoxy resin and a diethylenetriamine hardener obtained from the Photoelastic Division, Measurements Group, Inc., Raleigh, NC. After the epoxy cured, the glass was peeled off. Because the bonding between the aluminum film and the glass is rather weak, the aluminum coating is transferred onto the sample surface. The total thickness of the epoxy layer and the coating is just a couple of microns. Compared with the sample thickness, this layer is very thin and will not affect the deformation state inside the specimen.

The specimen was mounted in a drop weight tower (Dynatup-8100A) with a $200 \mathrm{~kg}$ free-falling weight, which is able to provide impacts at low to intermediate loading rates. After the impact, intensive stress waves emanate from the loading point and propagate toward the stationary crack. Stress fields are thus built up surrounding the crack tip and when the intensity of the stress field reaches a certain level, the stationary crack 
starts to propagate dynamically along the fiber direction. To record the CGS images during the experiment, a rotating mirror high-speed camera (Cordin model 330A) is used with a Spectra-Physics Argon-ion pulse laser (model 166) as the light source. The duration of the laser pulse is about $30 \mathrm{nsec}$, which is able to produce a sharp interference pattern even for fast running cracks. The interframe time (controlled by the interval between pulses) is typically $1.5 \mu \mathrm{sec}$. The laser pulse is triggered by a strain gage mounted to the specimen that senses the impact. The composite specimen is supported by resting its lower corners on two very thin glass slides. It has been determined that these glass slides can only sustain $20 \mathrm{~N}$ of load before they break. This suggests that the bottom surface of the specimen can be considered traction free throughout the crack propagation event.

\section{Experimental Observations}

A sequence of high speed CGS interferograms from a three-point bend test of the unidirectional graphite/epoxy IM7/8551-7 composite specimen is shown in Figure 4. The nominal impact speed was $4 \mathrm{~m} / \mathrm{sec}$ and the time
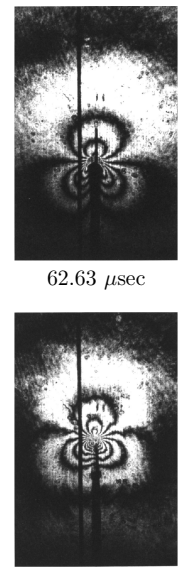

$70.98 \mu \mathrm{sec}$

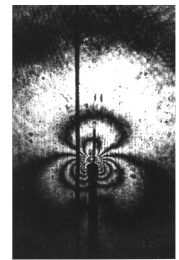

$64.30 \mu \mathrm{sec}$

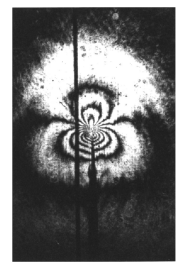

$72.65 \mu \mathrm{sec}$
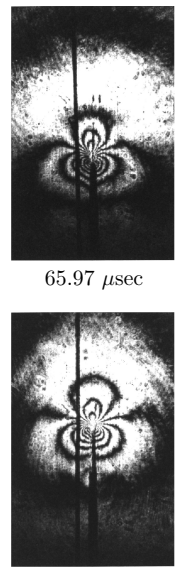

$74.32 \mu \mathrm{sec}$

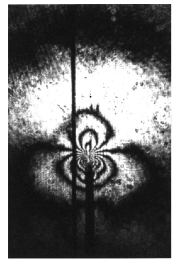

$67.64 \mu \mathrm{sec}$

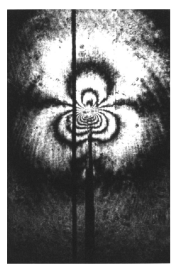

$75.99 \mu \mathrm{sec}$

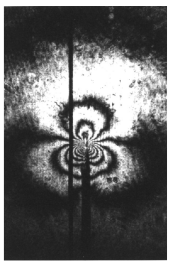

$69.31 \mu \mathrm{sec}$

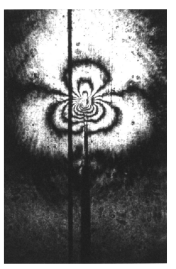

$77.66 \mu \mathrm{sec}$

Figure 4: Selected sequence of CGS interferograms from a unidirectional composite three-point bend dropweight tower test.

shown in Figure 4 is measured from the moment of impact. The interframe time for this specific test was $1.67 \mu \mathrm{sec}$. The two high-density gratings, shown in Figure 3, were perpendicular to the crack, so that the information regarding the quantity $\partial u_{3} / \partial \xi_{1}$ was obtained. The grating pitch was $p=0.025 \mathrm{~mm}$ and the distance between the two high-density gratings was set at $\Delta=46.5 \mathrm{~mm}$. The angular sensitivity of the CGS interferometer with these settings is about $0.015^{\circ}$ /fringe.

The first two interferograms shown in Figure 4 represent the CGS fringe patterns for the stationary crack. The size of the fringe pattern surrounding the stationary crack tip characterizes the intensity of the near-tip stress field. At a time close to $t=65.97 \mu \mathrm{sec}$, the stress field surrounding the tip reached a level such that the stationary crack starts to propagate. Since the unidirectional composite specimen was loaded symmetrically and the deformation surrounding the tip of the stationary crack is predominantly mode-I, the growing crack remains mode-I and it propagates along the fiber direction. This is also because the strength of the graphite fibers is so high that the crack is constraint to propagate along the fibers. Meanwhile, as the crack propagates dynamically through the material, the size of the fringe loop and the number of fringes change indicating the evolution of the intensity of the stress field surrounding the growing crack tip. One also observes that after crack initiation, stress waves emanate from the growing crack tip. They are visible in Figure 4 as discrete kinks of the CGS fringes. Such discrete kinks do not appear in the CGS fringe patterns associated with the stationary crack as shown in the first two interferograms in Figure 4.

The crack-tip location at each moment can be identified from the CGS fringe pattern. Consequently, the crack-tip velocity can be calculated based on the variation of crack length as a function of time. For the threepoint bend test of the unidirectional graphite/epoxy composite specimen shown in Figure 4, the variation of the amount of crack growth $\Delta a$ and the variation of crack-tip speed $\dot{a}$ as functions of time $t$ are presented in Figure 5. After initiation, the crack tip accelerates rapidly. In a very short period of time $(\sim 6 \mu \mathrm{sec})$ the 


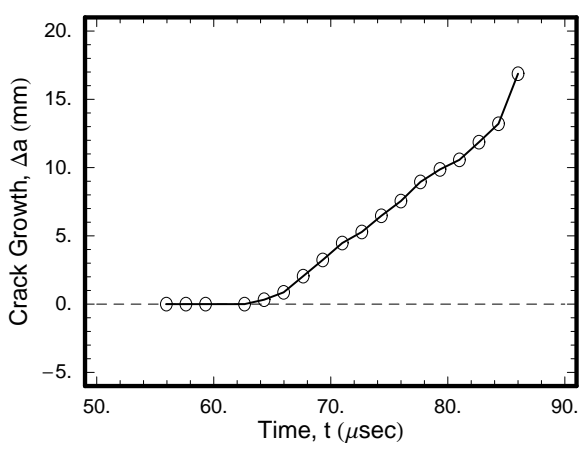

(a)

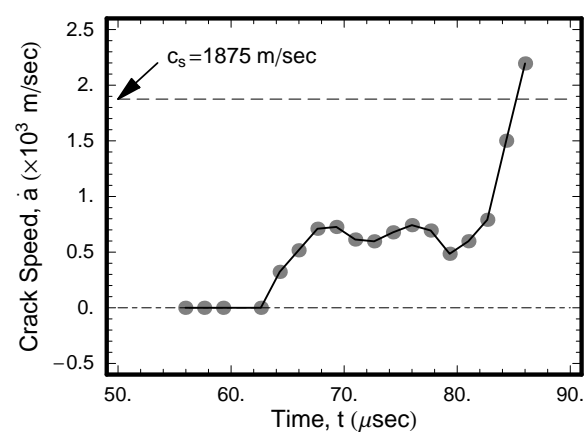

(b)

Figure 5: (a) Amount of crack growth $\Delta a$ and (b) crack-tip speed $\dot{a}$ as functions of time $t$ for the experiment shown in Figure 4.

crack reaches a relatively stable state and the crack-tip velocity oscillates around $650 \mathrm{~m} / \mathrm{sec}$. This relatively stable state lasts about $15 \mu \mathrm{sec}$ after which the crack tip accelerates again. Near the end of the experiment, the crack-tip velocity was well above the shear wave speed of the unidirectional graphite/epoxy composite, shown as the dashed line in Figure 5(b). Once the crack-tip velocity becomes larger than the shear wave speed of the material, the crack is said to propagate in the intersonic regime. Since the analysis presented in the previous section is only valid when the crack-tip speed is between zero and the shear wave speed of the material or when the crack propagates in the subsonic regime, the experimental results for the dynamic fracture toughness measurement were obtained only for moments when the crack-tip velocity was below the shear wave speed of the composite.

\section{Dynamic Fracture Toughness Measurement}

To obtain the dynamic stress-intensity factors at the moving crack tip, the photographs of the CGS fringe patterns were first digitized. According to the analyses of Rosakis and Ravi-Chandar [13] and Krishnaswamy et al. [14], data points must be chosen at a distance from the crack tip greater than half of the specimen thickness, to avoid the influence of three-dimensional effects. A least squares scheme was used to fit the experimental data to the first equation in (35) since the information represented by the CGS interferograms shown in Figure 4 relate specifically to the quantity $\partial u_{3} / \partial \xi_{1}$. The dynamic stress-intensity factors $K_{\mathrm{I}}$ and $K_{\mathrm{II}}$, at each moment, are therefore obtained. The energy release rate or the dynamic fracture toughness $\mathcal{G}$ is then calculated through (28) at each moment.

Both the variation of the dynamic stress-intensity factors, $K_{\mathrm{I}}$ and $K_{\mathrm{II}}$, and the variation of the dynamic fracture toughness $\mathcal{G}$, as functions of time after impact $t$, are presented in Figure 6. Although the single-edge-

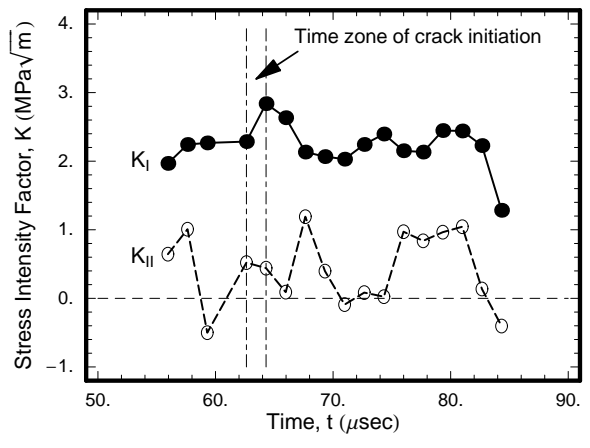

(a)

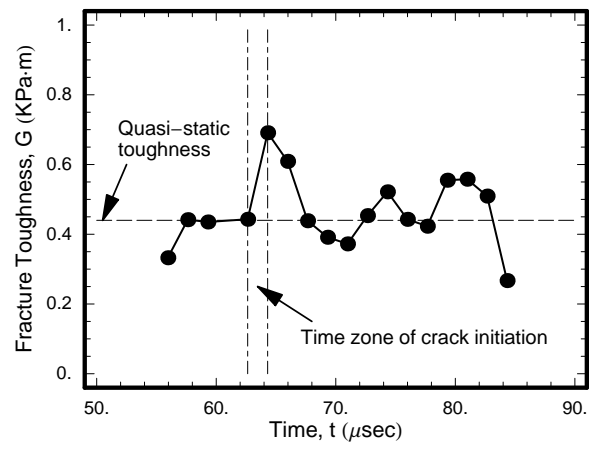

(b)

Figure 6: Variations of (a) the dynamic stress-intensity factors, $K_{\mathrm{I}}$ and $K_{\mathrm{II}}$, and (b) the dynamic fracture toughness $\mathcal{G}$ as functions of time $t$ for the experiment shown in Figure 4.

crack three-point bend specimen was loaded in a symmetric manner, due to the slight misalignment of the 
loading point and the center of the specimen we see the presence of the mode-II stress-intensity factor $K_{\mathrm{II}}$ in Figure 6(a) as open circles. Nevertheless, one can see that the intensity of the stress field surrounding the stationary and the subsequent moving crack is dominated by the mode-I stress-intensity factor (solid circles in Figure 6(a)). Meanwhile, due to the difficulty of identifying the exact moment of crack initiation, the uncertainty in time of initiation is presented by the two vertical dotted-dash lines in both plots in Figure 6. For the same composite material, Liu et al. [5] measured the initiation toughness under the quasi-static loading conditions using the same CGS technique described in this study. The quasi-static initiation toughness is represented by the horizontal dashed line in Figure 6(b), and here we do observe the so-called loadingrate dependency of the dynamic crack initiation toughness. The loading rate at the moment of dynamic crack initiation can be characterized by the quantity $\dot{K}_{\mathrm{I}}$, and from Figure $6(\mathrm{a})$, we can estimate that $\dot{K}_{\mathrm{I}} \sim$ $3.3127 \times 10^{5} \mathrm{MPa} \cdot \sqrt{\mathrm{m}} / \mathrm{sec}$.

Finally, we plotted the dynamic fracture toughness $\mathcal{G}$ against the crack-tip velocity $v$ in Figure 7 . For

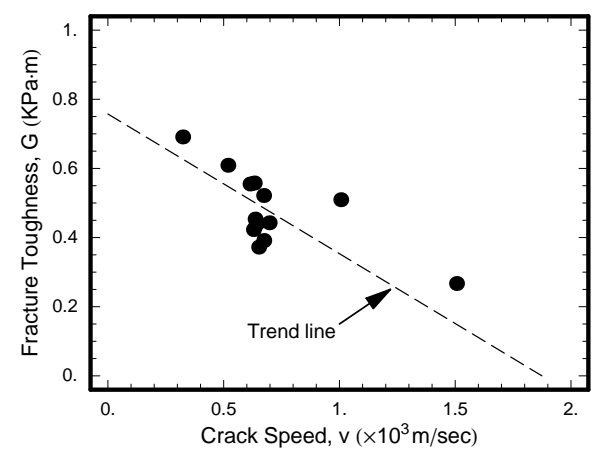

Figure 7: Dynamic fracture toughness $\mathcal{G}$ versus crack-tip velocity $v$ for the experiment shown in Figure 4.

a brittle crack propagating dynamically in an elastic solid, the most common criteria governing the crack growth are the generalizations of Irwin's critical stress-intensity factor criterion and Griffith's critical energy release rate criterion [10]. According to the generalized Griffith criterion, the crack will propagate in such a way that the crack-tip energy release rate is always equal to the dynamic fracture energy of the material, or the energy required to generate new material surfaces under dynamic conditions. The dynamic fracture energy, or the dynamic fracture toughness, of the material represents the resistance of the material to crack advance. The dynamic fracture toughness is assumed to be a material property and can only be determined from experiments. It is also believed that the dynamic fracture toughness, as a material property, depends on conditions under which the material is fractured, for example the temperature and the rate at which the material is separating. Therefore, the variation of the dynamic fracture toughness as a function of the crack-tip speed, which characterizes the rate of material separation, can be treated as a criterion that governs crack tip propagation if the other environmental conditions are fixed. This relationship has been measured for many different materials. The plot shown in Figure 7 indicates that there is a relationship between the dynamic fracture toughness and the crack-tip velocity for the unidirectional graphite/epoxy composite. The plot also seems to suggest that the dynamic fracture toughness is a decreasing function of the crack-tip velocity. This decreasing trend between the dynamic fracture toughness and the crack-tip speed has been observed in bimaterial systems where the crack propagates dynamically along the interface [15] and was justified based on more fundamental criteria. For a fiber-reinforced composite material, the interfacial toughness between the fiber and the matrix is believed to control the ultimate toughness of the bulk material. As a result, the overall trend observed in Figure 7 is consistent with the findings of Lambros and Rosakis [15] for bimaterial systems. In Figure 7, we plot a straight trend line which drops to zero at the shear wave speed of the unidirectional graphite/epoxy composite. However, reaching a definite conclusion regarding the exact relationship between the dynamic fracture toughness and the crack-tip velocity requires more experimental data, especially results that cover the entire subsonic regime.

\section{CONCLUDING REMARKS}

In this investigation, we studied the process of dynamic crack propagation in the IM7/8551-7 unidirectional graphite/epoxy composite using the optical CGS technique in conjunction with high-speed photography. The 
CGS interferometric fringe patterns were analyzed based on an elastodynamic analysis of crack propagation in orthotropic solids. The dynamic stress-intensity factors were extracted from the CGS fringe pattern analysis and the dynamic fracture toughness of the composite material was determined. We have demonstrated that the CGS optical technique is a useful tool to assess the resistance of a fiber-reinforced composite against dynamic crack propagation. However, it has to be pointed out that the analysis we used in this study is based on the assumption of a steady-state condition. From the experimental observations, one can see that the crack-tip velocity is only approximately constant during a short period of time. Although our experimental results indicate that the dynamic fracture toughness is a decreasing function of the crack-tip speed within the subsonic regime, more experimental measurements are needed to establish the exact relationship between these two quantities. Another issue that we did not address in this investigation, is the effect of mode-mixity on the dynamic fracture toughness. It has been found that the fracture toughness, of the same composite material studied in this paper, is a strong function of crack-tip mode-mixity under quasi-static loading conditions [4]. Since in most unidirectional fiber reinforced composites, due to the high strength of the fibers, the crack is often constrained to propagate along the fiber direction only. Thus, the mode-mixity at the moving crack tip might play an important role in determining the dynamic fracture toughness of the material, as we have seen in the interfacial crack propagation in bimaterial systems [15].

\section{Acknowledgements}

This research was supported financially by the U.S. Department of Energy, Defense Programs, Technology Transfer Initiative Program. AJR also acknowledges the financial support from the Office of Naval Research through a grant to Caltech (ONR Grant N00014-95-1-0453). The authors would like to thank Mr. R.W. Ellis of Los Alamos National Laboratory, for the assistance in conducting the dynamic CGS experiments presented in this study.

\section{References}

1. Davies P. and Benzeggagh M.L., 1989, "Interlaminar mode-I fracture testing," Application of Fracture Mechanics to Composite Materials, edited by K. Friedrich, Elsevier, New York, pp.81-112.

2. Chai, H., 1990, "Interlaminar shear fracture of laminated composites," International Journal of Fracture, 43, pp.117131.

3. Yoon S.H. and Hong C.S., 1990, "Interlaminar fracture toughness of graphite/epoxy composite under mixed-mode deformations," Experimental Mechanics, 30, pp.234-239.

4. Liu, C., Huang, Y., Lovato, M.L. and Stout, M.G., 1997, "Measurement of the fracture toughness of a fiber-reinforced composite using the Brazilian disk sample geometry," International Journal of Fracture, 87, pp.241-263.

5. Liu, C., Rosakis, A.J., Ellis, R.W. and Stout, M.G., 1998, "A study of the fracture behavior of unidirectional fiberreinforced composite using Coherent Gradient Sensing (CGS) interferometry," International Journal of Fracture, 90, pp.355-382.

6. Wu, K.-C., 1989, “On the crack-tip fields of an dynamically propagating crack in an anisotropic solid,” International Journal of Fracture, 41, pp.253-266.

7. Yang, W., Suo, Z. and Shih, C.F., 1991, "Mechanics of dynamic debonding," Proceedings of the Royal Society of London, A433, pp.679-697.

8. Stroh, A.N., 1958, "Dislocations and cracks in anisotropic elasticity," Philosophical Magazine, 3, pp.645-646.

9. Irwin, G.R., 1960, "Fracture mechanics," Structural Mechanics: Proceedings of the 1st Symposium on Naval Structural Mechanics, edited by J.N. Goodier and N.J. Hoff, Pergamon Press, New York, pp.557-591.

10. Freund, L.B., 1990, Dynamic Fracture Mechanics, Cambridge University Press, Cambridge.

11. Hutchinson, J.W. and Suo, Z., 1992, "Mixed mode cracking in layered materials," Advances in Applied Mechanics, edited by J.W. Hutchinson and T. Wu, 29, pp.63-191.

12. Rosakis, A.J., 1993, "Two optical techniques sensitive to gradients of optical path difference: The method of caustics and the coherent gradient sensor (CGS)," Experimental Techniques in Fracture, edited by J. Epstein, 3, Chapter 10, pp.327-425.

13. Rosakis, A.J. and Ravi-Chandar, K., 1986, "On crack-tip stress state: An experimental evaluation of threedimensional effects," International Journal of Solids and Structures," 22, pp.121-134.

14. Krishnaswamy, S., Tippur, H.V. and Rosakis, A.J., 1992, "Measurement of transient crack tip deformation fields using the method of coherent gradient sensing," Journal of the Mechanics and Physics of Solids, 40, pp.339-372.

15. Lambros, J. and Rosakis, A.J., 1995, "Development of a dynamic decohesion criterion for subsonic fracture of the interface between two dissimilar materials," Proceedings of the Royal Society of London, A451, pp.711-736. 